\title{
Development of Low-Cost Autonomous Emergency Braking System (AEBS) for an Electric Car
}

\author{
Mochammad Ariyanto \\ Department of Mechanical Engineering \\ Diponegoro University \\ Semarang, Indonesia \\ ari_janto5@yahoo.co.id
}

\author{
Gunawan D. Haryadi \\ Department of Mechanical Engineering \\ Diponegoro University \\ Semarang, Indonesia \\ gunawan_dh@yahoo.com
}

\author{
M. Munadi \\ Department of Mechanical Engineering \\ Diponegoro University \\ Semarang, Indonesia \\ muna_096@yahoo.com
}

\author{
Rifky Ismail \\ Department of Mechanical Engineering \\ Diponegoro University \\ Semarang, Indonesia \\ ismail.rifky@gmail.com
}

\author{
Zulfa Hendra \\ Department of Mechanical Engineering \\ Diponegoro University \\ Semarang, Indonesia \\ hendra.zulfa@gmail.com
}

\begin{abstract}
This paper presents the development of a low-cost Autonomous Emergency Braking System (AEBS) that can assist the driver or take over the electric car prototype for slowing down and breaking the car at the low-speed condition. An Actuator of AEBS is selected from DC motor that widely found on the market. The proposed AEBS is placed under the hood of the car. The AEBS will pull the brake pedal by using a Bowden cable. Ultrasonic sensor will be used for measuring the distance between the electric car and obstacle. On-off control will be developed for controlling the AEBS in breaking the electric car when the obstacle is approaching. After the AEBS prototype has been built, it will be employed in the electric car and tested for the automatic braking system. Based on the test results, the proposed AEBS can slow down and brake the car before colliding with the obstacle. The AEBS system will be activated when the distance between the car and the obstacle is less than $3.6 \mathrm{~m}$. Based on the test result, AEBS can slow down and brake the electric car before colliding with the obstacle.
\end{abstract}

\section{Keywords-low-cost, electric car, AEBS, braking system}

\section{INTRODUCTION}

One of the most common causes of accidents in the road is when a vehicle driver does not focus in driving the car like using a cell phone, tired body condition, having many thoughts and others while the vehicle in front of the car braking suddenly. The driver does not have time to brake or slow down the vehicle so that the vehicle will collide the car in front of him/her. To reduce the number of vehicle accidents due to these factors, it is important to develop the automatic control system that can make braking or slowdown of the car/vehicle when the driver is not in the ready state [1], [2].

The Autonomous Emergency Braking System (AEBS) is an automatic braking technology that combines sensors and brake controls to prevent collisions. Some automatic braking systems can prevent collisions at all, but most are designed to reduce vehicle speed before crashing into the front of the car. Because high-speed accidents are more likely to be fatal than low-speed collisions, automatic braking systems can save lives and reduce the amount of property damage that occurs during a crash. Some of these systems provide braking assistance to the driver, and the others can activate the emergency braking without input from the driver.

Some researchers use various sensors to measure the obstacle in front of the car. Laser scanner sensor has been used to measure the distance by research study [3]. The research shows that the laser scanner sensor can measure the distance needed for braking and slowing down the vehicle. Junkwang Kim [4] developed the Autonomous Emergency Braking (AEB) System based on commercial radar. The AEB can successfully work with the long braking distance from $3.3 \mathrm{~m}$ to $31.7 \mathrm{~m}$. Radar has been widely used in AEB research study [5]-[7]. Another widely used AEB sensor in the commercial car is LIDAR [8]. The AEBS based LIDAR has successfully reacted to emerging objects in time.

In this study, we proposed a low-cost braking system called Autonomous Emergency Braking System (AEBS). The AEBS will take over the car when the human driver is not ready to brake the car. This active braking safety system is usually installed on luxury cars. In this study, the developed AEBS uses the ultrasonic sensor for measuring the distance between the electric car and the obstacle, as well as DC motor as an actuator which will pull the brake pedal. Before the automatic braking work, the system will give a warning stage of alarm sounds and displays on the LCD. For controlling of AEBS, modified on-off control will be employed in this research. The proposed AEBS will be implemented on the prototype of an electric car for autonomous braking at the lowspeed test.

\section{Design of Autonomous EMERGENCY BRAKING System} (AEBS)

In the development of Autonomous Emergency Braking System (AEBS), the first thing to do is to model a brake pedal pulling mechanism that can move the brake pedal when the driver does not respond. This mechanism is attached to the brake pedal lever which will be connected via Bowden cable and pulled by with a high torque DC motor.

Power window DC motors are usually used to drive the car window with a large torque of $\pm 3030 \mathrm{kgf} . \mathrm{cm}$ and a gearbox inside it. Power window motors are also very easy to get, and the price is not too expensive. Based on these considerations, it was decided to become the actuator in the proposed AEBS. TABLE I summarize the specification of the selected power window motor.

Gear is chosen to transmit power from the motor to the shaft which will rotate the roller to pull the brake pedal. The manufacturing is also not too difficult. In addition to continuing power, the gear can also increase torque by increasing the number of teeth. In the power window, the 
motor has a gear so that it is easier to transmit with gear as well. Bowden cable is selected because it can withstand large pulling forces, strong and durable. The installation process in the AEBS is quite difficult. This Bowden cable will be used as a link between the brake pedal and the roller in AEBS which will pull or extend the brake pedal in the car. Low-cost ultrasonic sensors are employed in the proposed AEBS for measuring the frontal obstacle, even though the range is much shorter than using LIDAR or radar. But the cost or price is much cheaper compared to radar or LIDAR sensors.

After all parts of the AEBS components are designed and manufactured, the assembly is carried out. First is an assembly of DC motors, gearboxes, and rollers. The sensor and case infrared assembly are carried out then combine the two parts. This part is used to measure how much Bowden cable wire that has been pulled by DC motor in AEBS. In this research, infrared SHARP Sensor GP2Y0A41SK0F was used to measure the length of the wire pulled by AEBS. The calibration of the infrared sensor is conducted by the analog to digital (ADC) value measured by a microcontroller as shown in Fig. 1. DC motor assembly, gearbox, and the wire of Bowden cable can be seen in Fig. 2.a, while assembly case of the infrared sensor can be seen in Fig. 2.b. The complete final assembly of all brake pedal drive mechanisms that are ready to be attached to an electric car is shown in Fig. 2.c.

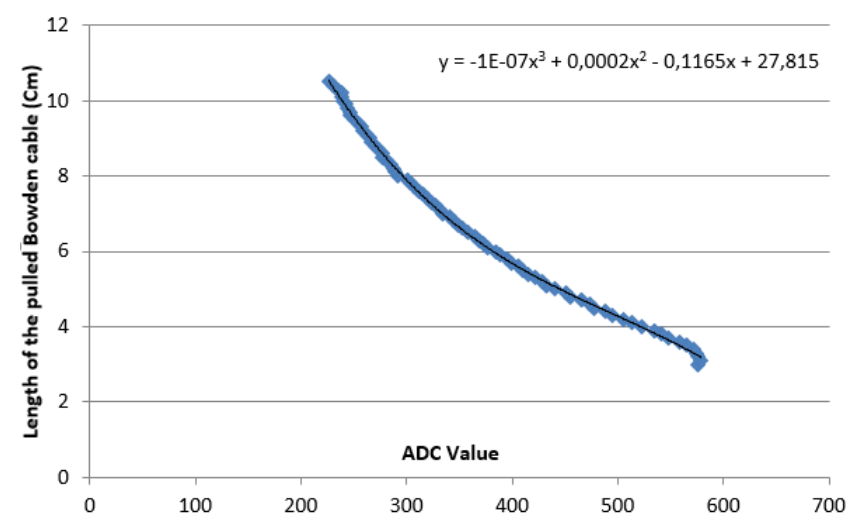

Fig. 1. Calibrated of the infrared sensor.

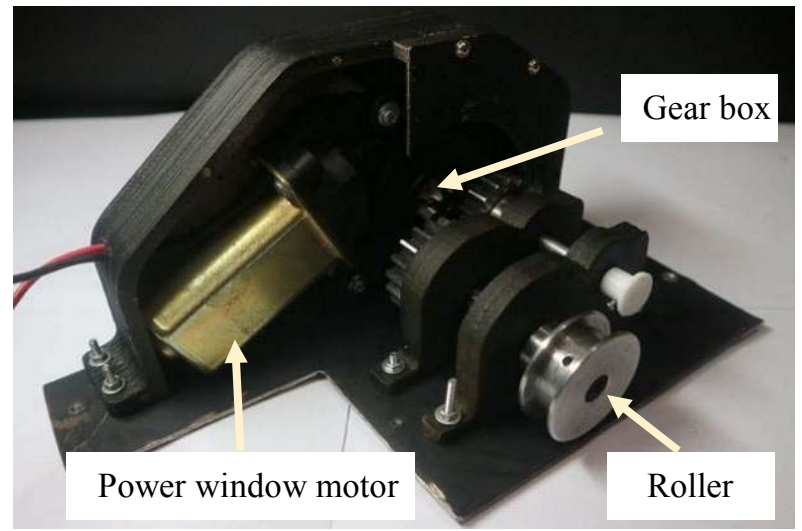

(a)

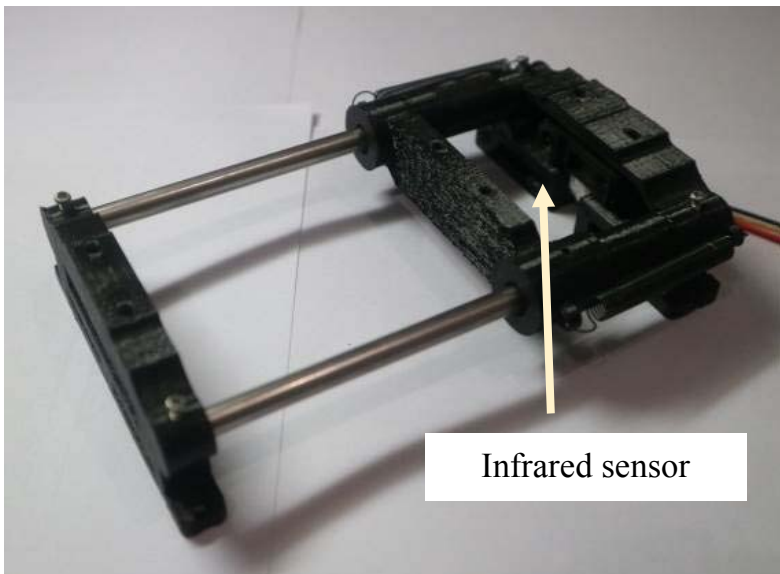

(b)

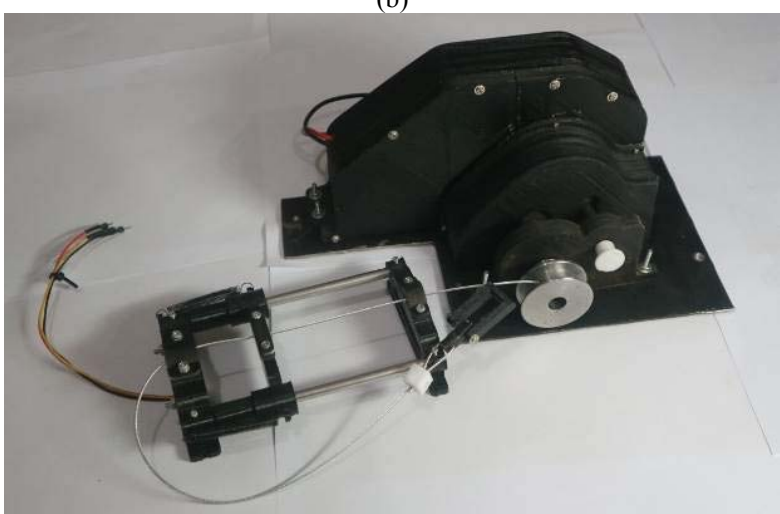

(c)

Fig. 2. Proposed ARBS prototype (a) Assembly of DC motor, gearbox, and roller, (b) Assembly case of the infrared sensor, (c) Brake pedal drive mechanism.

TABLE I. TECHNICAL SPECIFICATION OF THE UTILIZED POWER WINDOW MOTOR

\begin{tabular}{|l|c|}
\hline \multicolumn{1}{|c|}{ Parameter } & Value \\
\hline Working Voltage & $12 \mathrm{~V}$ \\
\hline Torque & $30 \mathrm{Kgf.cm}$ \\
\hline Maximum torque & $85 \mathrm{Kgf.cm}$ \\
\hline Play Speed & $70 \mathrm{rpm}$ \\
\hline Current & $7 \mathrm{~A}$ \\
\hline
\end{tabular}

\section{AEBS CONTROL}

Bang-bang control also called two-step control or on-off control, this control is widely used to control the plant that has an upper and lower limit. To get the maximum braking process on the brake pedal pulling mechanism, it needs to be taken into account when the DC motor stops rotating or pulls the pedal. For example, to make optimum braking, the pedal must move by $5 \mathrm{~cm}$, automatically by giving the command to pull the cable from AEBS by $5 \mathrm{~cm}$ and continue to maintain the condition/position. The proposed control in AEBS and the implementation algorithm developed in MATLAB/Simulink can be shown in Fig. 3 and Fig. 4 respectively.

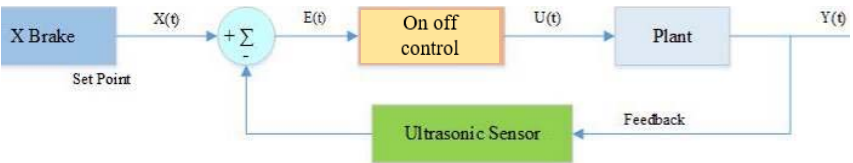

Fig. 3. Diagram block of On-off control in proposed AEBS 


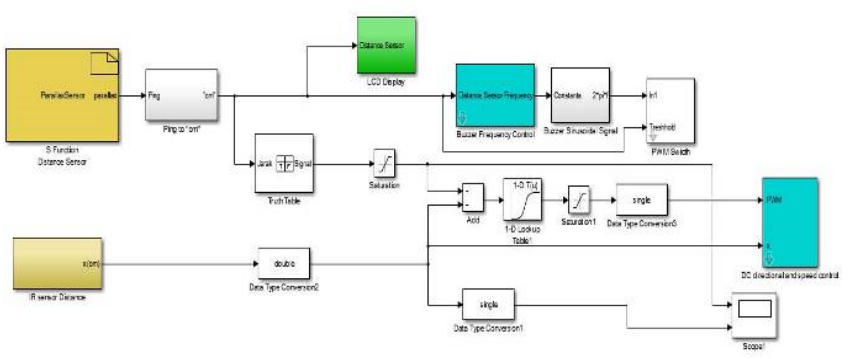

Fig. 4. Proposed AEBS On-off control in a Simulink environment.

Infrared sensor has been calibrated first as shown in Fig. 1 , so that it can directly provide an output of the length of the pulled brake pedal (X). The expected control is when the input command is in the form of a cable length that has been pulled by AEBS (X). With the feedback from the infrared sensor, the DC motor will adjust the length of the stretch or pull of the cable as much as the input command given. To give the logic of motion to the motor, it needs the corresponding pulse width modulation (PWM) value to produce a relatively small steady state error. The schematic of AEBS can be seen in Fig. 5.

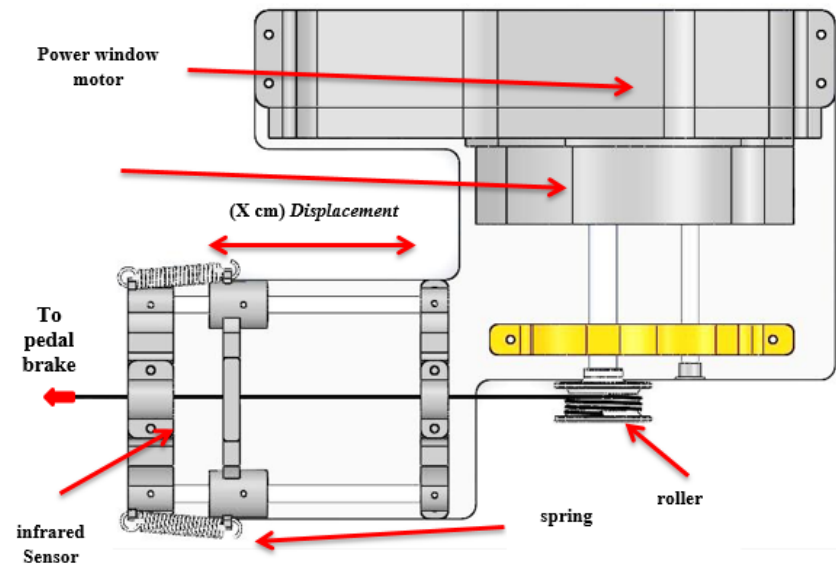

Fig. 5. AEBS for pulling the brake pedal.

\section{RESUlt AND DisCUSSION}

In this section, the AEBS prototype is tested in a real environment at low speed. Tests on the control that have been developed are carried out with two variations; the first without using a load and then by attaching a load which means that the AEBS is connected to the brake pedal in the electric car. The performance control test result with input in the form of a step is shown in Fig. 6.

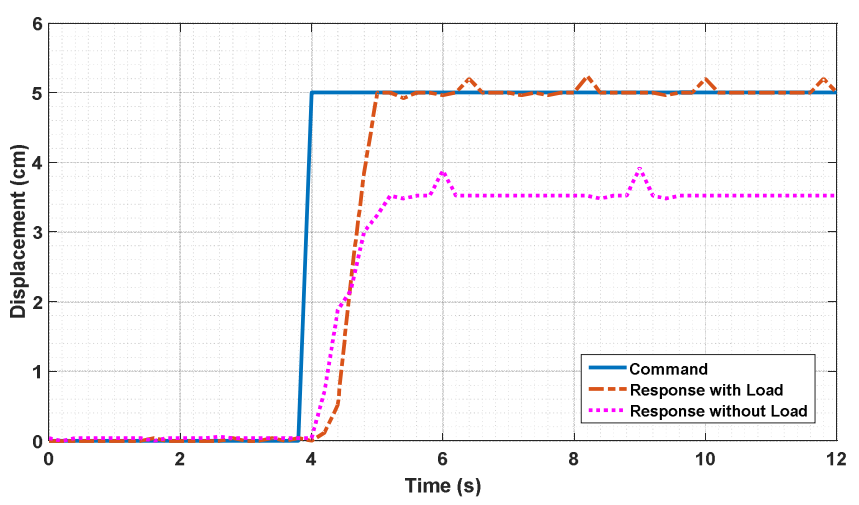

Fig. 6. Trajectory following command response
Based on Fig. 6, the steady-state error resulted without connected to the brake pedal is less than the error when the AEBS is connected to the brake pedal. It can occur because the DC motor needs more torque to pull the brake pedal. The performance of the proposed controller with step input is summarized in TABLE II.

TABLE II. PERFORMANCE OF THE PROPOSED ON-OFF CONTROL

\begin{tabular}{|l|c|c|c|}
\hline \multicolumn{1}{|c|}{ Performance } & $\begin{array}{c}\text { Without } \\
\text { Load }\end{array}$ & $\begin{array}{c}\text { With } \\
\text { Load }\end{array}$ & Unit \\
\hline Time constant $(\tau)$ & 0,47 & 0,37 & $\mathrm{~s}$ \\
\hline Rise time $\left(\mathrm{T}_{\mathrm{r}}\right)$ & 0,61 & 0,68 & $\mathrm{~s}$ \\
\hline Settling time $\left(\mathrm{T}_{\mathrm{s}}\right)$ & 1,89 & 1,48 & $\mathrm{~s}$ \\
\hline Delay time $\left(\mathrm{T}_{\mathrm{d}}\right)$ & 0,38 & 0,27 & $\mathrm{~s}$ \\
\hline Steady-state error $\left(\mathrm{e}_{\mathrm{ss}}\right)$ & 0,00 & 15 & $\mathrm{~mm}$ \\
\hline
\end{tabular}

Fig. 7 shows a graph of the AEBS response when pulling Bowden cable without being connected to the brake pedal in the electric car. Based on the figure, the proposed control can follow the input command with the maximum resulting in steady state-error less than $0.5 \mathrm{~cm}$.

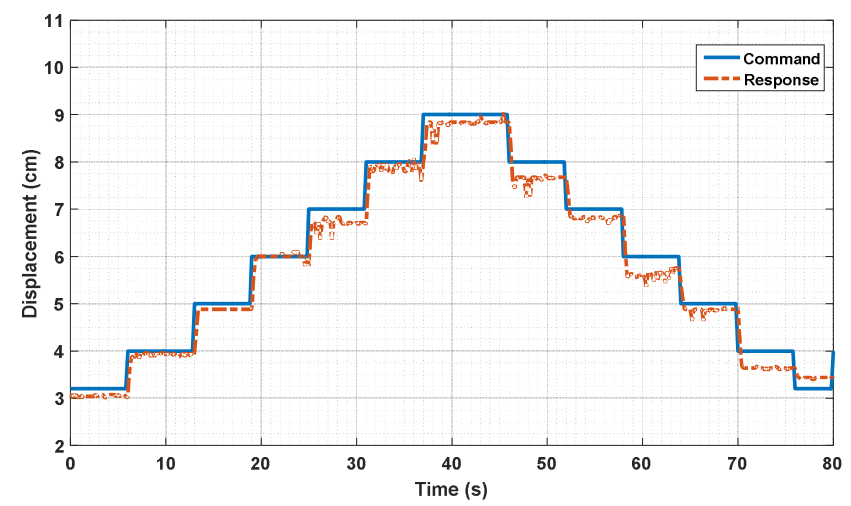

Fig. 7. Trajectory following command response

Installation of AEBS mechanical and electrical hardware in an electric car is carried out before testing for crash avoidance or emergency braking in the front of the car. The attachment of the autonomous emergency braking and the ultrasonic sensor is presented in Fig. 8.a. and Fig. 8.b. respectively. These parts are placed outside the electric car. The connection of the AEB to the pedal is shown in Fig. 8.c. while the LCD and the buzzer are placed on the dashboard of the car. These parts are attached inside the car.

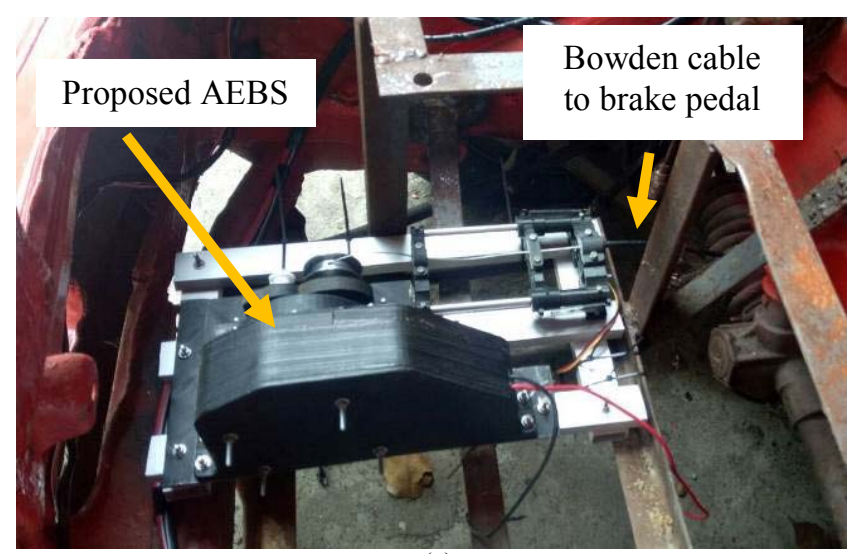

(a) 


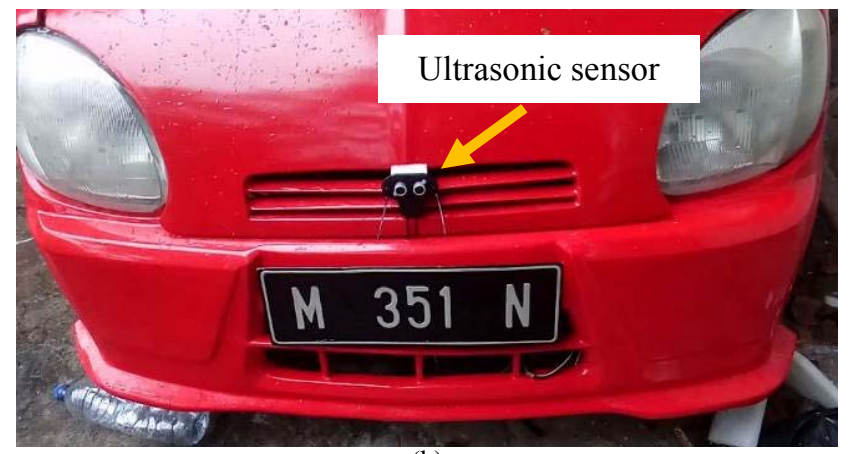

(b)

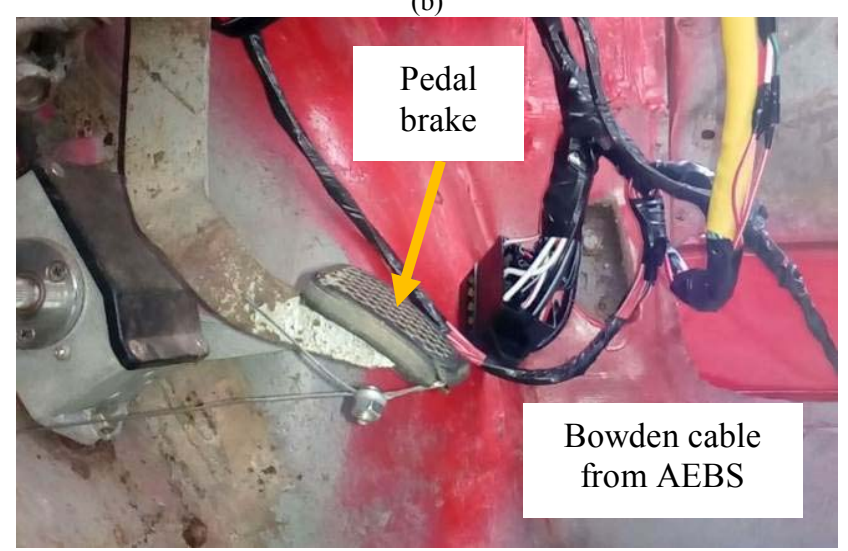

(c)

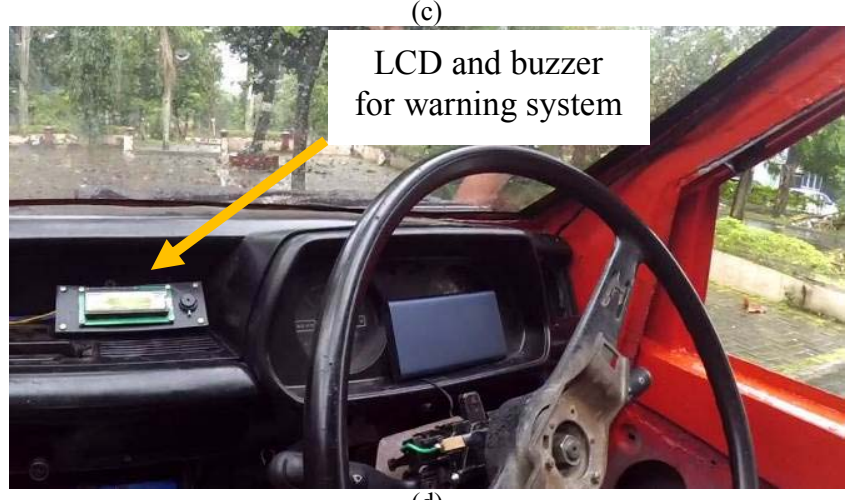

(d)

Fig. 8. Implementation of the proposed AEBS on electric car prototype. (a) Attachment of the AEBS under the hood car, (b) Attachment of the ultrasonic sensor, (c) Connection of brake pedal to AEBS via Bowden cable, (d) LCD and buzzer placement.

This test is aimed to find out the response to the proposed AEBS, whether AEBS can carry out emergency braking on time and in accordance with the input given when there is an obstacle in front of the car. The car used in the test is an electric car in which hydraulic brake is installed like other commercial cars. While the obstacle used is made of plywood wood with a width and height of $130 \mathrm{~cm} \times 80 \mathrm{~cm}$ made in such a way that ultrasonic sensors can be detected the obstacle. The car and the obstacle for the emergency test can be seen in Fig. 9.a. and Fig. 9.b.

The maximum range of obstacle that can be detected by the ultrasonic sensor is $4 \mathrm{~m}$. Because of the limitation in the reading the range of the obstacle, the emergency braking is set to active when the distance between the car and the obstacle is less than $3.6 \mathrm{~m}$. Another warning setting on the AEBS is summarized by TABLE III.
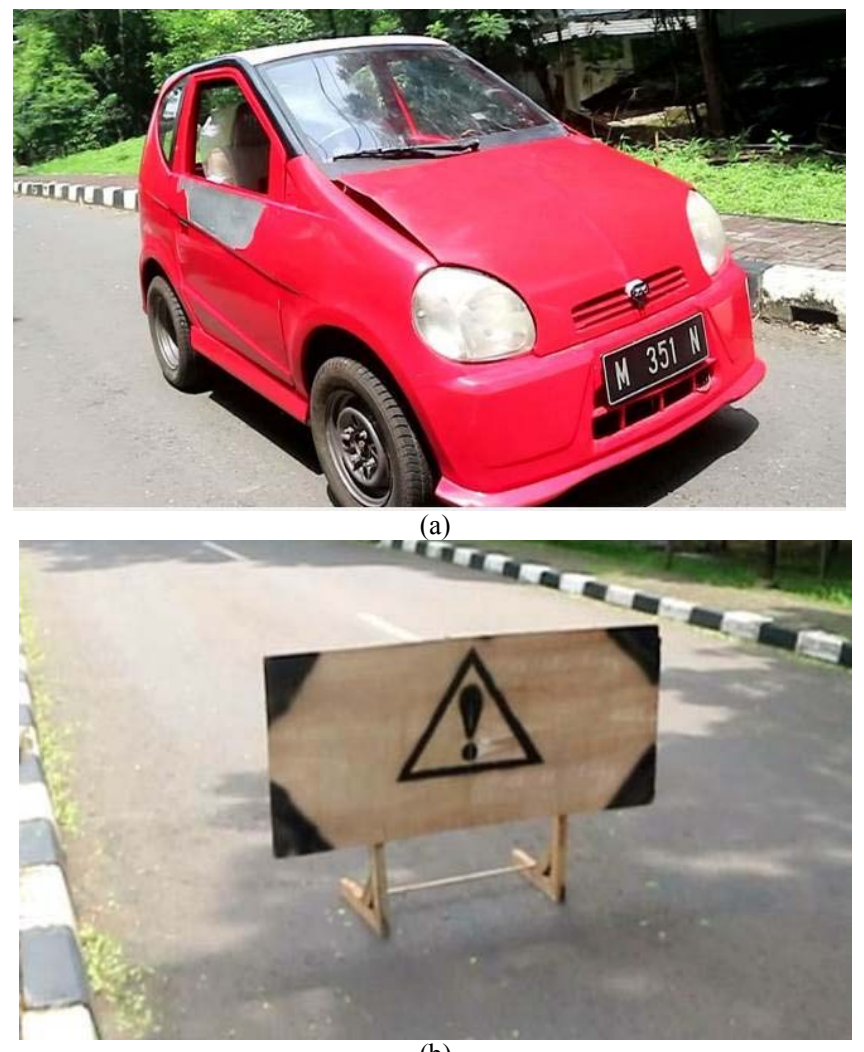

(b)

Fig. 9. Emergency braking test (a) Electric car prototype, (b) obstacle.

TABLE III. DISTANCE SETTING FOR AEBS

\begin{tabular}{|c|c|}
\hline AEBS response & $\begin{array}{c}\text { Sensor Distance } \\
\text { to Obstacle }\end{array}$ \\
\hline Warning system on LCD & $370 \mathrm{~cm}$ \\
\hline Warning system on alarm buzzer & $370 \mathrm{~cm}$ \\
\hline Autonomous braking active & $360 \mathrm{~cm}$ \\
\hline
\end{tabular}

In an emergency braking test, the distance between the car and the obstacle is $15 \mathrm{~m}$. The car runs with an average speed of $5 \mathrm{~km} / \mathrm{h}$ or $1.4 \mathrm{~m} / \mathrm{s}$. The car moves closer to the obstacle, and then AEBS is activated before the distance between the car and obstacle is less than $3.6 \mathrm{~m}$. When the obstacle distance and the car reach $3.7 \mathrm{~m}$, the warning system is activated and displayed the distance to the LCD on the car dashboard. The sequence images of the emergency braking test can be seen in Fig. 10.

From the results of the emergency braking test at low speed, it was found that at 30 seconds the car had entered a distance of less than $360 \mathrm{~cm}$ from the obstacle then the system gave a command to immediately pull the brake pedal to slow down and stop the car. The car can successfully stop at a sufficient distance.

\section{CONCLUSION}

In this study, the low-cost AEBS was successfully developed and built for an electric car. The steady-state error generated without the brake pedal is less than the error when the AEBS is connected to the brake pedal. Based on the result of an emergency braking test, the proposed AEBS can slow down and brake the electric car before colliding with the obstacle.

In the future study, the higher torque DC motor will be used to replace the current power window motor to reduce the 
steady-state error in autonomous braking. The ultrasonic sensor will be replaced by LIDAR or radar in order to increase the distance range that can be detected by these sensors. More dynamic model and advanced control will be developed and implemented such as artificial intelligence in order to smooth the braking response.

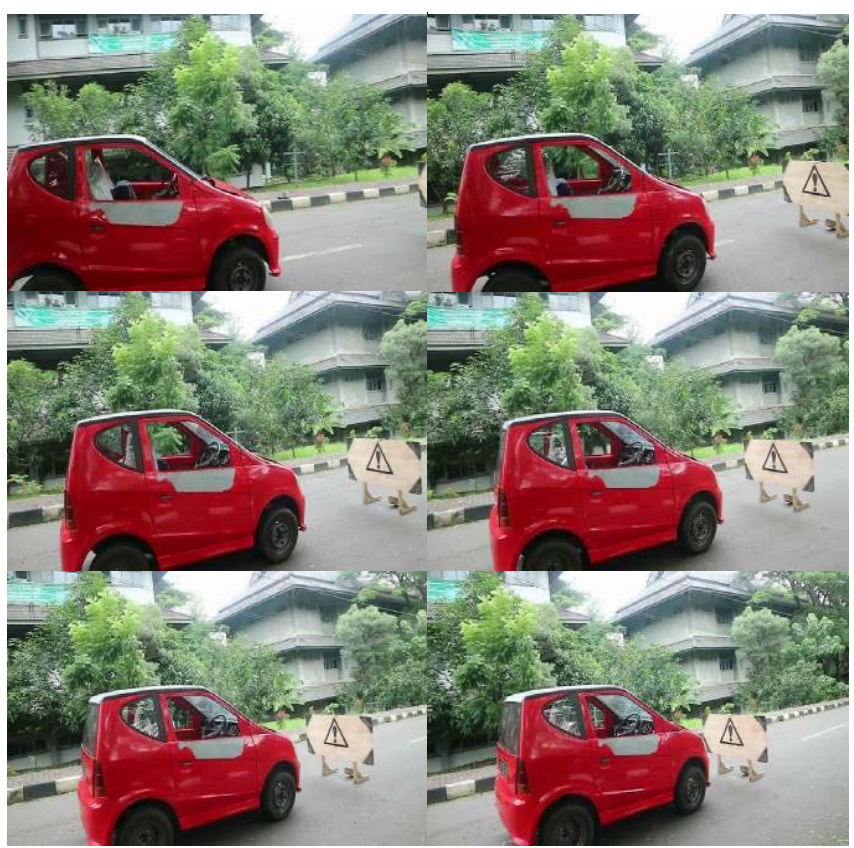

Fig. 10. Sequence images of automatic braking test

\section{ACKNOWLEDGMENT}

The authors gratefully acknowledge partial funding support from the United States Agency International
Development (USAID) under the Sustainable Higher Education Research (SHERA) program.

\section{REFERENCES}

[1] H. Kopetz and S. Poledna, "Autonomous Emergency Braking: A System-of-Systems perspective," in 2013 43rd Annual IEEE/IFIP Conference on Dependable Systems and Networks Workshop (DSN-W), 2013, pp. 1-7.

[2] D. H. Lee, S. K. Kim, C. S. Kim, and K. S. Huh, "Development of an autonomous braking system using the predicted stopping distance," Int. J. Automot. Technol., vol. 15, no. 2, pp. 341-346, Mar. 2014.

[3] Ping-Fan Jin, Jung-Hyun Kim, and Jung-Ha Kim, "Design of unmanned vehicle advanced braking system using smart motor," in 2015 12th International Conference on Ubiquitous Robots and Ambient Intelligence (URAI), 2015, pp. 167-171.

[4] J. Kim, W. Y. Jung, S. Kwon, and Y. Kim, "Performance Test of Autonomous Emergency Braking System Based on Commercial Radar," in 2016 5th IIAI International Congress on Advanced Applied Informatics (IIAI-AAI), 2016, pp. 1211-1212.

[5] H. Kim and B. Song, "Vehicle recognition based on radar and vision sensor fusion for automatic emergency braking," in 2013 13th International Conference on Control, Automation and Systems (ICCAS 2013), 2013, pp. 1342-1346.

[6] A. Wedel and U. Franke, "Monocular Video serves RADAR-based Emergency Braking," in 2007 IEEE Intelligent Vehicles Symposium, 2007, pp. 93-98.

[7] J. Andersson, "Developing Advanced Emergency Braking Systems at Scania - MATLAB \&amp; Simulink," www.mathworks.com, 2016. [Online]. Available: https://www.mathworks.com/company/newsletters/articles/devel oping-advanced-emergency-braking-systems-at-scania.html. [Accessed: 23-Nov-2018].

[8] J. Wallner, T. Tang, and M. Lienkamp, "Development of an Emergency Braking System for Teleoperated Vehicles Based on Lidar Sensor Data," in Proceedings of the 11th International Conference on Informatics in Control, Automation and Robotics, 2014, pp. 569-576. 